\title{
Comparison of 99mTc-DMSA Renal Scan and Power Doppler Ultrasonography for the Detection of Acute Pyelonephritis and Vesicoureteral Reflux
}

\author{
Hee Jung Bae, M.D. ${ }^{1}$ \\ Yong-Hoon Park, MD., Ph.D. ${ }^{1}$ \\ Jae Ho Cho, M.D. ${ }^{2}$ \\ Kyung Mi Jang, M.D.'
}

Department of Pediatrics ${ }^{1}$, College of Medicine, Yeungnam University, Daegu, Korea, Department of Radiology ${ }^{2}$, College of Medicine, Yeungnam University, Daegu, Korea

Corresponding author:

Kyung Mi Jang, M.D.

Department of Pediatrics, College of

Medicine, Yeungnam University, 170

Hyunchungno, Nam-gu, Daegu 42415, Korea

Tel: +82-53-620-3533

Fax: +82-53-629-2252

E-mail:fortune001j@gmail.com

Received: 14 September 2018

Revised: 8 October 2018

Accepted: 10 October 2018
Purpose: Urinary tract infection (UTI) is one of the common infectious diseases in children. Several imaging modalities can be used to confirm the presence of acute pyelonephritis (APN). Among them the 99mTcdimercaptosuccinic acid renal scan (DMSA scan) is used as a gold standard for diagnosis. Ultrasonography technology is evolving. Therefore, in this study, we investigated the sensitivity and specificity of Power Doppler ultrasonography (PDU) compared to the results from the previous study.

Methods: There were 260 patients included in this study, aged between 1 and 12 months old. The patients were admitted to the Yeungnam University Medical Center between January 2008 and December 2015. All patients underwent both DMSA scan and PDU within 5 days of admission. Voiding cystourethrography (VCUG) was performed in 195 patients with abnormal DMSA scan or PDU.

Results: The diagnostic sensitivity of APN using PDU was $45.5 \%$ and specificity was $85.5 \%$ in 260 patients following detection of a defect on DMSA scan that was defined as APN. The diagnostic sensitivity and specificity of PDU for VUR were 65.5 $\%$ and $60.1 \%$, respectively. The diagnostic sensitivity and specificity of DMSA scan forVUR were $95.7 \%$ and $14.1 \%$, respectively.

Conclusion: PDU has a high specificity but low sensitivity, so there are limitations in using it to replace a DMSA scan for the diagnosis of APN in children. DMSA scan and PDU have different sensitivity and specificity in diagnosis of VUR, respectively. Therefore, we suggest that the sensitivity and specificity of each test can be helpful in diagnosing APN and VUR when used in conjunction.

Key words: 99mTc-DMSA, Power Doppler Ultrasonography, Pyelonephritis, Vesicoureteral Reflux

\section{Introduction}

Urinary tract infection (UTI) is one of the common bacterial infectious diseases in childhood. There are inherent difficulties in diagnosing infants because they frequently have nonspecific symptoms ${ }^{1}$. Upper UTI in some infants can cause hypertension and chronic renal damage ${ }^{2)}$. Therefore, rapid diagnosis and treatment are critical.

Using a 99mTc-dimercaptosuccinic acid renal scan (DMSA scan), acute pyelonephritis (APN) can be diagnosed at the acute phase, and renal scarring can be diagnosed at 3-6 months following infection ${ }^{3,4)}$. However, DMSA scans
This is an open-access article distributed under the terms of the Creative Commons Attribution Non-Commercial License (http:// creativecommons.org/licenses/by-nc/4.0/) permits unrestricted non-commercia medium, provided the original work is properly cited. \\ Pediatric Nephrology}


involve exposure to radiation as well as requiring intravenous drug administration and sedation. The use of computed tomography (CT) has a high sensitivity for APN diagnosis; however, it involves a high dose of radiation and requires administration of a contrast agent ${ }^{5)}$. Therefore, a DMSA scan is preferred in clinical practice. Magnetic resonance imaging (MRI) can also be used to diagnose APN, but it is not suitable for practical clinical applications because of time consuming and high cost ${ }^{6,7)}$. Power Doppler ultrasonography (PDU) is a noninvasive technique that can be used to monitor the size and shape of the kidney ${ }^{8-10)}$. The presently used technique of color doppler ultrasonography can provide an image in color only when the speed of the blood flow is higher than a specific speed and does not expose a vessel adequately if the angle between the insonation beam and vessel is sharp or if the vessel is in a region at a distance from the transducer. PDU was developed to overcome these disadvantages. This technique displays color on the basis of the total strength of the Doppler signal, thus, making it easy to examine weak blood flow. In addition, interference from the angle is relatively small; therefore, PDU is more sensitive than color doppler ultrasonography ${ }^{11)}$.

About a decade ago, the author's hospital reported the sensitivity and specificity of PDU in the diagnosis of APN ${ }^{12)}$. Ultrasonography technology is evolving. Therefore, in this study, we investigated the sensitivity and specificity of PDU compared to the results from the previous study and the patients with VUR.

\section{Material and methods}

A total of 260 patients were included in this study, aged between 1 and 12 months old. The patients were admitted to the Yeungnam University Medical Center between January 2008 and December 2015 and diagnosed with a UTI for the first time. All colony-forming counts were higher than $5 \times 10^{4}$ in urine culture with suprapubic aspiration or catheterization. Blood samples were performed for all the patients before administration of antibiotics, including leukocyte counts, erythrocyte sedimentation rate (ESR), C-reactive protein (CRP) and blood culture. Children with an underlying disease, including known congenital urogenital anomalies (e.g. renal agenesis, renal hypoplasia, renal dysplasia, polycystic kidneys, multicystic dysplastic kidneys and duplex ureters) were excluded from this study. All patients underwent both a DMSA scan and PDU after the fever subsided. The tests were performed within 5 days of admission.

DMSA scan images were obtained by intravenous injection of $111 \mathrm{MBq}$ Tc-99mTc-DMSA and the kidneys were scanned with continuous rotation images after 3 hours. After correcting the background, left and right activity fractions were calculated for each kidney and an uptake of $45-55 \%$ of the total kidney activity was presumed normal. When the labeled DMSA showed one or more local or diffuse intake reductions in the kidney or an overall decrease in the enlarged kidney, it was judged to be abnormal. PDU images were obtained on both sides, in prone and supine positions using variable-frequency curved transducers on a LOGIQ7 ultrasound imaging system (General Electric Company). Gray-scale sonography was also performed, and PDU was judged to be abnormal when there was no or decreased flow in the kidney parenchyma compared with other areas of the same depth in the same kidney. VCUG was performed in 195 patients with suspected VUR following abnormal findings on the DMSA scan or PDU. Statistical analysis was performed using the Mann-Whitney test and the Fisher's exact test using SPSS version 22.0 (IBM).

\section{Results}

Of the 260 patients included in the study, 191 were diagnosed with a cortical defect in their DMSA scan and were diagnosed with APN. Of the 191 APNs diagnosed, the majority, 137 (72\%) were boys and 54 (28\%) were girls $(P<0.05)$. Escherichia coli (E. coli) was cultured in most of the patients $(173,91 \%)$. When we compared the patients with positive findings and those with negative findings in the DMSA scan, leukocyte counts, ESR, and CRP were significantly higher in the positive group (Table 1).

Of the 191 patients with a defect on their DMSA renal scan, there were 87 patients with PDU defects, and among 69 patients with a normal DMSA scan, 59 also showed normal findings in PDU. When the DMSA scan was used as the reference for APN, the diagnostic sensitivity of PDU was $45.5 \%$, specificity was $85.5 \%$, positive predictive value 
was $89.6 \%$, and negative predictive value was $36.2 \%$. The diagnostic sensitivity of PDU for 520 left and right kidneys was $42.1 \%$, specificity was $96.5 \%$, positive predictive values were $90.6 \%$ and negative predictive values were $67.8 \%$. (Table 2).

Among the 260 patients included in the study, 195 children underwent VCUG. VUR was found in 47 (24.1\%) children and was found in 61 (15.6\%) kidneys. Of the 47 patients with VUR, there were 45 patients with abnormal finding in DMSA scan, and 31 patients with a defect in PDU. There were 31 patients with abnormalities in both tests. Of the 61 kidneys, international VUR grade 1 was found in $3(0.8 \%)$, grade 2 in 9 (2.3\%), grade 3 in 17 (4.4\%), grade 4 in 24 (6.2 $\%)$, and grade 5 in 8 (2.0\%). The diagnostic sensitivity and specificity of the DMSA scan for VUR were $95.7 \%$ and 14.1 $\%$, respectively. The diagnostic sensitivities and specificities of PDU for VUR were $65.5 \%$ and $60.1 \%$, respectively (Tables 3).

The 191 patients with abnormal findings in their DMSA scan were followed up for 6 months. Except for 82 patients who were lost to follow up at 6 months, 109 patients performed DMSA scan. The cortical defect was improved in 73 (67\%) of the patients, however, a cortical defect was still observed in 36 (33\%). At 6 months of follow-up, abnormalities were detected in the initial PDU in 38 (52.1\%) of 73 patients in whom the cortical defect disappeared and in 20 (55.5\%) of 36 patients in whom a cortical defect was still present. Patients with cortical defect had slightly more initial PDU abnormalities but, there were no significant differences. The 36 patients with a persistent cortical defect at 1 year performed a follow-up DMSA scan. Except for 22 patients who were lost to follow up at 1 year, 14 patients per- formed DMSA scan. Two patients (14.3\%) out of 14 patients were improved and a cortical defect was still observed in $12(85.7 \%)$ children. Of the 12 patients in whom a cortical defect was still present at 1 year, the kidney size decreased in 1 patient and renal uptake was reduced in 3 patients. At 2 years of follow-up, two patients still had cortical defect and renal scarring, but there were no clinical symptoms in the patients with renal scarring.

\section{Discussion}

In children, UTI is one of the common infectious diseases and is known to have a prevalence of $3 \%$ in girls and $1 \%$ in boys ${ }^{13)}$. However, it is difficult to diagnose, as symptoms are often nonspecific ${ }^{14)}$. Renal scarring, one of the major complications of APN, has been reported to occur in appro-

Table 2. Diagnostic and Predictive Values of PDU Comparison with DMSA

\begin{tabular}{lcc}
\hline & $\ln 260$ patients & $\ln 520$ Kidneys \\
\hline Sensitivity & $45.5 \%$ & $42.1 \%$ \\
Specificity & $85.5 \%$ & $96.5 \%$ \\
Positive predictive value & $89.6 \%$ & $90.6 \%$ \\
Negative predictive value & $36.2 \%$ & $67.8 \%$ \\
\hline
\end{tabular}

Abbreviations: PDU, power doppler ultrasonography; DMSA, 99mTcdimercaptosuccinic acid.

\begin{tabular}{lcc}
\multicolumn{3}{l}{ Table 3. Diagnostic Values of DMSA and PDU for VUR } \\
\hline DMSA scan & PDU \\
\hline Sensitivity & $95.7 \%$ & $65.5 \%$ \\
Specificity & $14.1 \%$ & $60.1 \%$ \\
\hline
\end{tabular}

Abbreviations: DMSA, Tc-99m dimercaptosuccinic acid; PDU, power doppler ultrasonography; VUR, vesicoureteral reflux.

Table 1. Demographics and Laboratory Data of the Patients

\begin{tabular}{|c|c|c|c|}
\hline & \multicolumn{2}{|c|}{ DMSA scan } & \multirow{2}{*}{ Total } \\
\hline & Normal & Abnormal (APN) & \\
\hline Number of patients & 69 & 191 & 260 \\
\hline Mean age(month) & $4.8 \pm 2.5$ & $5.3 \pm 2.3$ & $5.2 \pm 2.4$ \\
\hline $\operatorname{Sex}(M: F)$ & $4.8: 1$ & $2.5: 1$ & 2.9:1 \\
\hline $\mathrm{WBC}(/ \mu \mathrm{L})$ & $13,698.4 \pm 5,136.9$ & $16,877.5 \pm 6,429.7^{*}$ & $15,593.0 \pm 6,209.4$ \\
\hline $\operatorname{ESR}(\mathrm{mm} / \mathrm{h})$ & $21.4 \pm 18.9$ & $41.4 \pm 29.6^{*}$ & $36.1 \pm 28.5$ \\
\hline CRP (mg/L) & $2.37 \pm 2.20$ & $6.58 \pm 5.89^{*}$ & $5.46 \pm 5.48$ \\
\hline Abnormal finding in PDU & 10 & 87 & 97 \\
\hline
\end{tabular}


ximately $64 \%$ of children, and this renal damage can lead to end-stage renal disease ${ }^{15,16)}$. Therefore, early diagnosis and treatment is especially important to prevent complications. Many imaging studies have been used to diagnose pediatric APN, and several studies have been conducted to evaluate usefulness of specific techniques ${ }^{9}$. Pathophysiologically, the blood flow of renal parenchyma is reduced by vasoconstriction of peripheral arterioles in the vascular phase of APN and the damaged segment can be detected by DMSA scan, CT, or PDU ${ }^{15,17,18)}$.

According to the Clinical Practice Guideline for the Diagnosis and Management of Initial UTI in Febrile Infants and Children 2 to 24 Months proposed by the American Academy of Pediatrics in 2011, noninvasive conventional renal ultrasonography is recommended as a primary imaging test for pediatric patients with urinary tract infection to detect anatomical abnormalities. Renal ultrasonography is typically used to diagnose hydronephrosis, renal abscess, and anomalies in the urinary system ${ }^{19)}$. In addition, the sensitivity of PDU for detecting ischemic injuries in blood vessels in the kidney, including the interlobar and arcuate arteries, is high. However, its sensitivity for detecting renal parenchymal invasion to diagnose pyelonephritis is low ${ }^{20)}$.

In 2007, we reported sensitivity and specificity values for the use of PDU for the diagnosis of APN. The age of the patients ranged from 1 month to 7 years old and the total number of patients was limited to 25. Compared with the previous study, we selected patients aged between 1 and 12 months, and the total number of patients was recruited 260. In 2007, the sensitivity and specificity for the use of PDU for diagnosis of APN were $38.1 \%$ and $50 \%$, respectively. In this study, the diagnostic sensitivity was $42.1 \%$, the specificity was $96.5 \%$, demonstrating that the sensitivity was similar but the specificity was increased compared with the results of 2007. The reasons for the between-study differences may be: First, the sample size was larger, and the patient age range was limited in the present study. Second, the test quality might have improved because the examiner had performed the procedure many times since the previous study and PDU equipment might be improved.

The sensitivity and specificity of the DMSA scan for VUR were $95.7 \%$ and $14.1 \%$, respectively, and the sensitivity of PDU was 65.5\%, which was lower than DMSA scan, but the specificity of PDU was $60.1 \%$ which was higher the
DMSA scan. In addition, of the 195 patients who underwent VCUG, 88 patients had abnormal findings with only a DMSA scan, and among them 14 patients (15.9\%) had VUR, whereas among 84 patients with abnormal findings on both DMSA scan and PDU, 31 patients (36.9\%) had VUR, and VUR was significantly higher in patients with abnormal findings in both tests $(P<0.05)$.

Considering VUR grades 1-3 as low-grade reflux and VUR grades 4 and 5 as high-grade reflux, 20 of 47 pediatric patients had low-grade reflux and 27 had high-grade reflux. For the diagnosis of low-grade VUR, the sensitivity and specificity of DMSA scan were $85.1 \%$ and $5 \%$, respectively and the sensitivity and specificity of PDU were $39.9 \%$ and $35.0 \%$, respectively. For diagnosing high-grade VUR, DMSA scan showed a sensitivity and specificity of $86.9 \%$ and 3.7\%, respectively, and PDU had a sensitivity and specificity of $42.9 \%$ and $33.3 \%$, respectively. Thus, there was no significant difference of the sensitivity or specificity in diagnosing low- and high-grade VUR. In diagnosing VUR, the DMSA scan showed high sensitivity but low sensitivity. In addition, both the DMSA scan and PDU test showed a significantly higher rate of VUR diagnosis than using a DMSA scan alone.

The limitation of the present study was that a comparison couldn't be made with pediatric patients showing abnormalities on PDU, because only those who showed abnormalities on the DMSA scan were followed up. Moreover, it was difficult to consider the exact values of DMSA and PDU because patients with urogenital anomaly were excluded. PDUs have many advantages, including no requirement for sedation, no exposure to radiation and no need for IV drugs. According to Jack S. Elder, about $50 \%$ of febrile UTI patients were positive on DMSA scan ${ }^{21)}$, however, in this study, 193 of 260 patients (73.5\%) were considered positive. In addition, the inflammatory markers such as leukocyte counts, ESR, and CRP were significantly higher in the positive group in DMSA scan. Moreover, in our study and our results of 2007, it seems that it is difficult to replace the DMSA scan with PDU because of its low sensitivity in the diagnosis of APN. But, PDU and DMSA scan show different sensitivities and specificities in the diagnosis of APN and VUR, respectively. Therefore, we suggest that the sensitivity and specificity of each test can be helpful in diagnosing APN and VUR when they are used in parallel. 


\section{Ethics statement}

This present study was reviewed and approved by the Institutional Review Board of Yeungnam University Hospital (IRB number YUMC 2018-07-042).

\section{Conflicts of interest}

No potential conflict of interest relevant to this article was reported.

\section{References}

1. Hoberman A, Chao HP, Keller DM, Hickey R, Davis HW, Ellis D. Prevalence of urinary tract infection in febrile infants. J Pediatr 1993; 123:17-23

2. Ditchfield MR, de Campo JF, Nolan TM, Cook DJ, Grimwood K, Powell HR, et al. Risk factors in the development of early renal cortical defects in children with urinary tract infection. AJR Am J Roentgenol 1994;162:1393-7.

3. Majd M, Rushton HG. Renal cortical scintigraphy in the diagnosis of acute pyelonephritis. Semin Nucl Med 1992;22:98-111.

4. Stokland E, Hellström M, Jacobsson B, Jodal U, Lundgren P, Sixt R. Early 99mTc dimercaptosuccinic acid (DMSA) scintigraphy in symptomatic first-time urinary tract infection. Acta Paediatrica 1996;85:430-6.

5. Dacher J, Boillot B, Eurin D, Marguet C, Mitrofanoff P, Le Dosseur P. Rational use of CT in acute pyelonephritis: Findings and relationships with reflux. Pediatr Radiol 1993;23:281-5.

6. Lonergan GJ, Pennington DJ, Morrison JC, Haws RM, Grimley MS, Kao TC. Childhood pyelonephritis: Comparison of gadoliniumenhanced MR imaging and renal cortical scintigraphy for diagnosis. Radiology 1998;207:377-84.

7. Papanicolaou N, Pfister RC. Acute renal infections. Radiol Clin North Am 1996;34:965-95.

8. Rubin JM, Bude RO, Carson PL, Bree RL, Adler RS. Power doppler US: A potentially useful alternative to mean frequency-based color doppler US. Radiology 1994;190:853-6.

9. Halevy R, Smolkin V, Bykov S, Chervinsky L, Sakran W, Koren A. Power doppler ultrasonography in the diagnosis of acute childhood pyelonephritis. Pediatric Nephrology 2004;19:987-91.

10. Eggli K, Eggli D. Color doppler sonography in pyelonephritis. Pediatr Radiol 1992;22:422-5.

11. Babcock D, Patriquin H, LaFortune M, Dauzat M. Power doppler sonography: Basic principles and clinical applications in children. Pediatr Radiol 1996;26:109-15.

12. Choi JY, Cho JH, Park YH. Power doppler sonography for the upper urinary tract infection in children. Yeungnam University Journal of Medicine 2007;24:179-85.

13. Clarke SE, Smellie JM, Prescod N, Gurney S, West DJ. Technetium99m-DMSA studies in pediatric urinary infection. J Nucl Med 1996;37:823-8.

14. Rushton HG. The evaluation of acute pyelonephritis and renal scarring with technetium 99m-dimercaptosuccinic acid renal scintigraphy: Evolving concepts and future directions. Pediatric Nephrology 1997;11:108-20.

15. Roberts JA. Etiology and pathophysiology of pyelonephritis. American Journal of Kidney Diseases 1991;17:1-9.

16. Jakobsson B, Berg U, Svensson L. Renal scarring after acute pyelonephritis. Arch Dis Child 1994;70:111-5.

17. Nosher JL, Tamminen JL, Amorosa JK, Kallich M. Acute focal bacterial nephritis. American Journal of Kidney Diseases 1988;11:3642.

18. Giblin JG, O'Connor KP, Fildes RD, Harkness B, Levin K, Newsome $J$ T, et al. The diagnosis of acute pyelonephritis in the piglet using single photon emission computerized tomography dimercaptosuccinic acid scintigraphy: A pathological correlation. J Urol 1993; 150:759-62.

19. Subcommittee on Urinary Tract Infection, Steering Committee on Quality Improvement and Management, Roberts KB. Urinary tract infection: Clinical practice guideline for the diagnosis and management of the initial UTI in febrile infants and children 2 to 24 months. Pediatrics 2011;128:595-610.

20. Johnson JR, Vincent LM, Wang K, Roberts PL, Stamm WE. Renal ultrasonographic correlates of acute pyelonephritis. Clinical Infectious Diseases 1992;14:15-22.

21. Jack S. Elder. Urinary Tract Infection. Nelson textbook of Pediatrics. 20th ed. Philadelphia: Elsevier, 2015:2556-62. 